\title{
Effective Method to Measure and Inspect the Hysteresis Loss of a Transformer
}

\author{
$1^{\text {st }}$ Arunava Chatterjee \\ Department of Electrical Engineering \\ Raghunathpur Govt. Polytechnic \\ Purulia, India. \\ arunava7.ju@gmail.com
}

\author{
$2^{\text {nd }}$ Sankar Das \\ Department of Electrical Engineering \\ Govt. College of Engineering and \\ Textile Technology \\ Berhampore, India. \\ sankar05ju@gmail.com
}

\author{
$3^{\text {rd }}$ Debashis Chatterjee \\ Department of Electrical Engineering \\ Jadavpur University \\ Kolkata, India. \\ debashisju@yahoo.com
}

\begin{abstract}
This paper presents a study on the significance of source side harmonics and their effects on transformers. Source side harmonics are typically present in power electronic sources which are commonly used in renewable applications. The continued outcome of source side harmonics is observed on the hysteresis curve of a transformer. Single-phase transformers are used in the proposed study to determine the effect of harmonics on magnetization and demagnetization cycles using an electronic operational amplifier-based integrator circuit. A technique is also presented for effectively storing and plotting the hysteresis curve from the measured data. After the hysteresis curve is obtained, it is compared with standard data and a conclusion is obtained from the results about the presence of harmonics in the source. The hysteresis curve is thus found without removing the transformer from operation. The study also proposes a modified hysteresis model for the transformer considering the effect of source harmonics. The proposed study is an effective tool for easy measurement and detection of harmonics. The MATLAB/Simulink based simulations with suitable experiments validate the proposed study.
\end{abstract}

Keywords-transformer, op-amp integrator, harmonics, hysteresis, piecewise-mixed model

\section{INTRODUCTION}

Source voltage and its harmonics effects in different electrical systems is an extensively studied subject. The study of harmonics and their consequence on power and distribution transformers is also well investigated theme. Usually, the effect of harmonics is movement of harmonic currents which causes upsurge in losses leading to increase in internal temperature of the transformer and potential faults [1]. Different studies are completed regarding upshot of harmonics in transformers. In [2], voltage harmonic effects on transformers are considered for high-power low voltage motors. Harmonic contamination for distribution transformers is extensively examined using modest open and short circuit tests [3]. This is a simple method for studying the effects though they require the transformer to be put out of action for such tests. Also, measurement and required modification of core losses in transformer is studied [4]. In this paper, mainly distorted supply voltage is measured. Estimation of core loss when the flux has fundamental and a single odd harmonic component in it is also studied in [5]. Different core sample materials are tested in the research and the core loss is estimated. Core loss calculation from low frequency quantities is done in [6] and shown to be operative for transformer laminations. Finite element-based hysteresis loop is calculated based on energetic hysteresis model. No-load loss estimation under sub-harmonics for power transformers is present in [7] using two-dimensional finite element model analysis. Under non-sinusoidal voltage supply, the core or the iron losses can be foreseen using improved formulae of core loss as prearranged in [8]. The same with modifications can be used for pulsed waveforms. Modified core loss for brushless DC (BLDC) machines are also predicted [9]. It is quite evident that core loss needs modifications when used for prediction of losses in case of supply with harmonics. In general, the transformer is not loaded at the rated value or it is functioned in less than rated value [10]. Transformer excitation current analysis under distorted supply is studied [11]. Approach for measurement and correction of losses under no-load conditions for distorted supply is also offered for power transformers [12]. For distribution transformer, harmonic impact is studied for no-load losses [13]. New methods for measurements of no-load losses for transformers is also studied [14].

In this paper, measurement and inspection of effect of source harmonics is made for single-phase transformers which are associated to power electronic source used frequently in renewable energy generation. The inverter with its high frequency switching produces non-sinusoidal voltage at output which may include harmonics. This study provides a decisive analysis on transformer operation when the source side comprises of these odd order harmonics. In this study, the supply is made distorted by including harmonics of odd order and the effect for the same is revealed on the hysteresis curve. By analyzing the hysteresis curve, an idea about the core loss of the transformer is also made using a proposed model which also can predict the loss with increased accuracy as shown.

\section{Proposed TeChNIQUE AND HARMONIC MODEL FOR TRANSFORMER WITH DISTORTED SUPPLY}

The proposed technique is targeted at finding the transformer hysteresis plot or the $B-H$ curve. Accordingly, some measurements are taken at different conditions. It is to be kept in mind that normally it is difficult to find the magnetic field intensity in the transformer core as well as the flux density. Thus, alternative method for measurement of different quantities is to be made for suitable analysis. The system connection along with the block diagram of the proposed scheme is shown in Fig.1. From Fig.1, after the signals are measured using the proposed technique, the same is used to construct the hysteresis curve. The curve is then compared with a standard hysteresis curve which is obtained from a transformer with similar ratings and without any source harmonics. The compared results are then checked for any deviation or error. If the error is greater than a tolerance value, the conclusion is made that the source has significant number of harmonics. Suitable measure is then taken to operate the transformer at a derated value so that the transformer windings current limit is not increased and the losses are minimized 
thereby effectively increasing the operating life of the transformer.

The transformer diagram for proposed measurement technique is shown in Fig.2. From Fig.2, the transformer magnetic field strength $H$ of the core material is found using (1),

$$
H=\left(N I_{m}\right) / l
$$

where, $N$ is number of turns and $l$ is the length of the core. $I_{m}$ is the magnetizing current. With $N$ and $l$ as constant values for a particular transformer with fixed core. For the precise measurement of $I_{m}$, a resistance $R_{x}$ is linked in series with the primary winding.

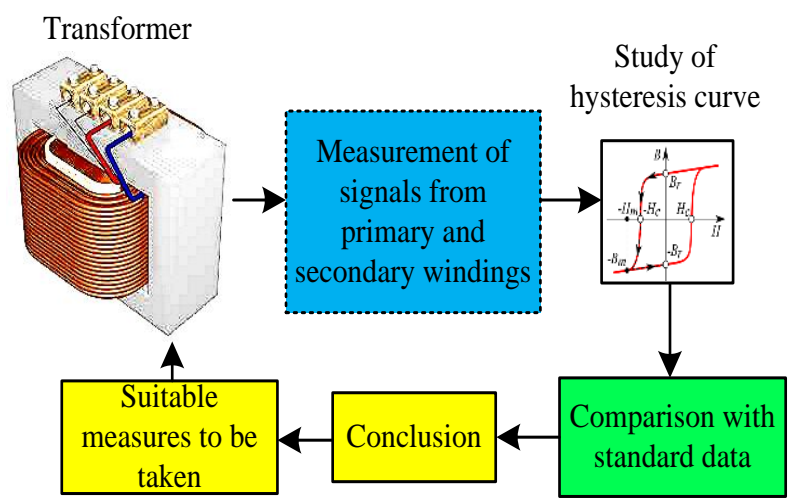

Fig. 1. Block diagram of proposed technique.

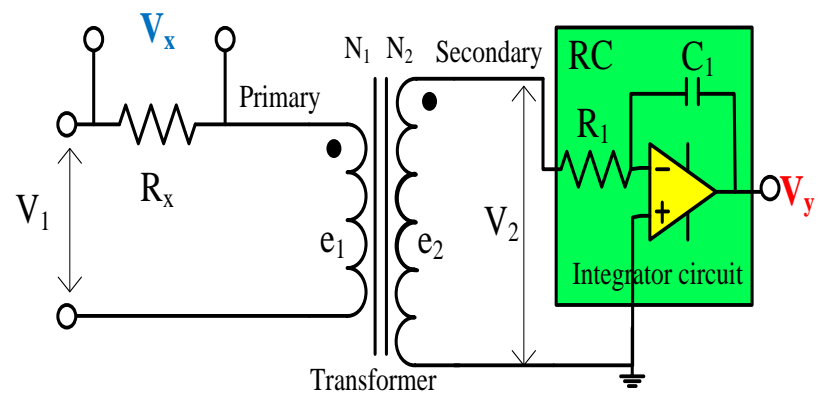

Fig. 2. Measurement technique.

The voltage across the resistor is proportional to the magnetizing current value which is again proportional to the core magnetic field strength.

$$
V_{x}=R_{x} I_{m}
$$

The same is derived in (2). For the hysteresis curve, the magnetic flux density is commonly found using [15],

$$
B=\phi / A
$$

where, $\phi$ is the magnetic flux (webers) and $A$ is the crosssectional area of the magnetic core. From (3), it can be observed that the magnetic flux density is directly proportional to the magnetic flux. The rate of change of flux is proportional to the induced emf. This is in turn proportional to the voltage at output. Thus, the magnitude of, induced emf $e_{2}$, is written from Faradays law (4) as [16],

$$
e_{2}=N_{2} \frac{d \phi}{d t}
$$

Also,

$$
\frac{1}{N_{2}} e_{2} d t=d \phi
$$

Integrating both sides of (5), one may obtain,

$$
\phi=\frac{1}{N_{2}} \int e_{2} d t
$$

Thus, flux is proportional to the integral of the secondary voltage. From (6), it is thus evident that the secondary terminal voltage can be integrated to form the required signal for flux density. For this purpose, an electronic operational amplifierbased integrator circuit is used.

The op-amp $R C$ integrator will act as a passive low-pass filter circuit and voltage across the capacitor can be measured as integrated output (7). From the integrator circuit,

$$
V_{y}=\frac{1}{R_{1} C_{1}} \int V_{2} d t
$$

\section{A. Parameter Choice for Measurement}

For finding the resistors $R_{x}$ and $R_{1}$ and capacitor $C_{1}$, the following process is used where the circuit components are selected on the basis physical quantities of the transformer. The same is derived from (8) for the resistor $R_{x}$, the number of primary turns be $N_{1}$, length of wire for primary $l$ with radius $r$,

$$
l=2 \pi r N_{1}
$$

Substituting value of (8) in (1),

$$
\begin{gathered}
H=I_{m} / 2 \pi r \\
\text { or, } H=I_{m} Z_{1}
\end{gathered}
$$

where, $Z_{1}(=1 / 2 \pi r)$ is a constant whose value depends on radius of primary wire. The value of $H$ can be expressed using $I_{m}$ from (2) and (10) as,

$$
H=V_{x} \frac{Z_{1}}{R_{x}}
$$

From (11) if, $Z_{1}=R_{x}$, then $H=V_{x}$. Once the primary side resistor value is chosen, the secondary side resistor and capacitor values are to be selected using the following technique.

For choosing resistor $R_{1}$ and capacitor $C_{1}$ in secondary side, from (3) and (6),

$$
B=\frac{1}{A N_{2}} \int e_{2} d t
$$

Taking Laplace transform on both sides of (12) with neglecting initial conditions, the flux density (13) is obtained as,

$$
B(s)=\frac{E_{2}(s)}{s}\left(\frac{1}{A N_{2}}\right)
$$

The transfer function of integrator network is given from (14),

$$
\frac{V_{y}(s)}{E_{2}(s)}=-\frac{1}{R_{1} C_{1} s}
$$

Thus from (13) and from (14),

$$
B(s)=-\left(\frac{R_{1} C_{1}}{A N_{2}}\right) V_{y}(s)
$$

If, $R_{1} C_{1}=A N_{2}$. Then, flux density (15) is simplified to (16) as,

$$
B(s)=-V_{y}(s)
$$

\section{B. Proposed Harmonic Model}

Magnetic flux density in core $B$ is pure integral of $V_{\mathrm{y}}$ with a multiplying factor of $\left(1 / A N_{2}\right)$. 
The core loss model of a magnetic material changes for presence of different harmonic frequencies as hysteresis curve depends on it. The core loss consists of hysteresis and eddy current losses. The hysteresis loss $P_{h}$ in watts using Steinmetz equation [17] is,

$$
P_{h}=k B_{m}^{x} f
$$

where $f$ is frequency of flux reversal and $k$ is hysteresis loss coefficient. Considering the harmonic frequencies, (17) is modified [9] for single-phase system as,

$$
P_{h}=k\left(B_{m 1}^{x} f_{1}+B_{m 3}^{x} f_{3}+B_{m 5}^{x} f_{5}+B_{m 7}^{x} f_{7}+\cdots+B_{m n}^{x} f_{n}\right)
$$

where subscripts, $3,5,7, \ldots, n$ corresponds to odd harmonic order of frequencies. For three-phase system, the triplen harmonics will be absent in per phase basis. This loss due to harmonics is increased in renewable systems employing wind power where an inverter is used for high frequency switching [18]-[20]. Due to the presence of odd harmonics in the supply, the hysteresis loss will change which will also be reflected in the model hysteresis curve. The hysteresis curve can be compared for a healthy running transformer with no supply harmonics to comprehend the operating behaviour of a transformer. Using (18), the hysteresis loss can be accurately calculated as shown in Table-I.

TABLE I. Simulated ANALYSIS OF SOURCE HARMONICS WITH THE PROPOSED MODEL

\begin{tabular}{|l|l|l|l|}
\hline Sl. No. & $\begin{array}{l}\text { Source } \\
\text { harmonics order }\end{array}$ & $\begin{array}{l}\text { Hysteresis loss (in } \\
\text { Watts) using } \\
\text { Steinmetz model }\end{array}$ & $\begin{array}{l}\text { Hysteresis loss } \\
\text { (in Watts) using } \\
\text { proposed model }\end{array}$ \\
\hline 1. & Fundamental only & 15 & 15 \\
\hline 2. & $\begin{array}{l}\text { Fundamental with } \\
3^{\text {rd }} \text { and } 5^{\text {th }}\end{array}$ & 32 & 37.5 \\
\hline 3. & $\begin{array}{l}\text { Fundamental with } \\
3^{\text {rd }}, 5^{\text {th }} \text { and } 7^{\text {th }}\end{array}$ & 51 & 52.7 \\
\hline
\end{tabular}

\section{RESULTS AND DISCUSSION}

The simulations are done using MATLAB/Simulink environment for single-phase transformers for the proposed technique. The rating of the transformer is chosen as 750VA, with $60 / 120 \mathrm{~V}, 50 \mathrm{~Hz}$ for experiment and simulation. The simulations are carried considering source harmonics and without source harmonics. The simulation waveform of supply voltage and the hysteresis curve are shown in Fig.3 when the supply is free from any harmonics.

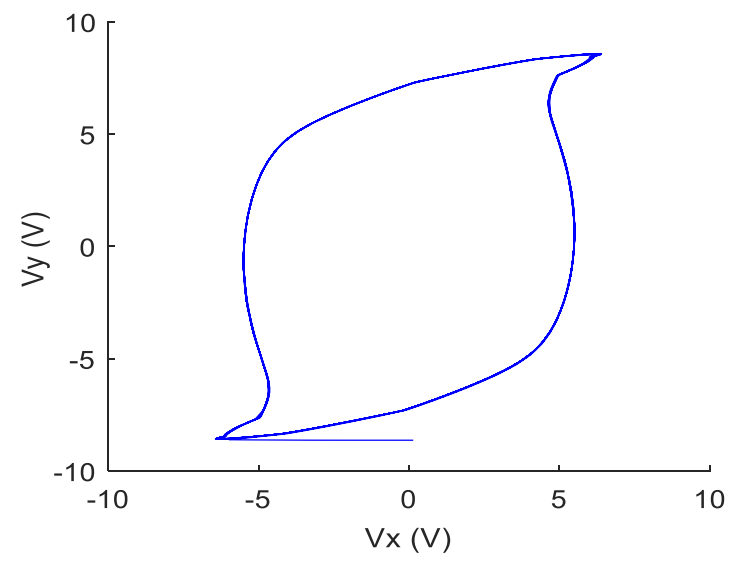

Fig. 3. Hysteresis curve for sinusoidal supply.
When the supply contains harmonics of $3^{\text {rd }}$ and $5^{\text {th }}$ order, the hysteresis curve is shown in Fig.4. The hysteresis curve in Fig.4 displays that when the supply contains lower order harmonics of substantial value, the collective effect of all the harmonic currents is observed in the hysteresis curve as curvatures and distortions. The smoothness of the hysteresis curve is entirely removed when the supply contains harmonics. Thus, the flux reversal is not smooth. In contrast to the supply without harmonics, the hysteresis curve shows smoother flux reversal.

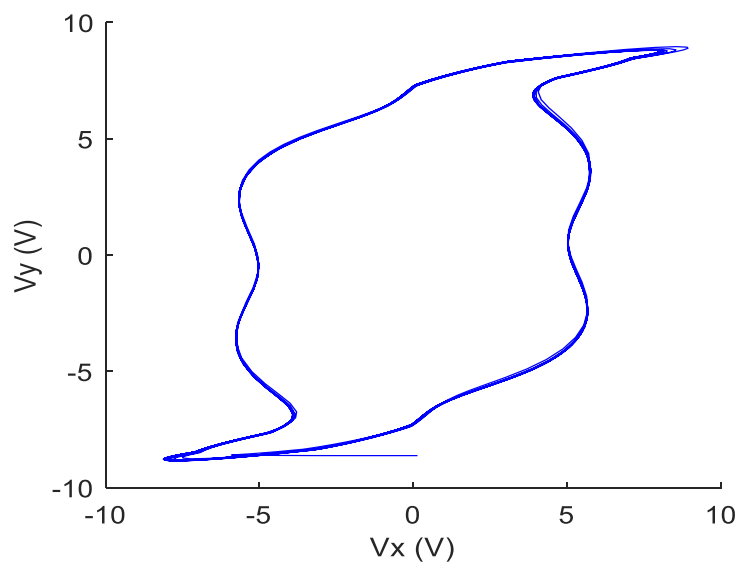

Fig. 4. Hysteresis curve for sinusoidal supply containing $3^{\text {rd }}$ and $5^{\text {th }}$ order harmonics.

It is clear than the hysteresis waveforms obtained has unusual curvatures than a normal hysteresis curve when the supply is strictly sinusoidal. This can be mathematically verified and analysed using standard data with modified curve fittingbased technique. The experimental setup is shown in Fig.5.

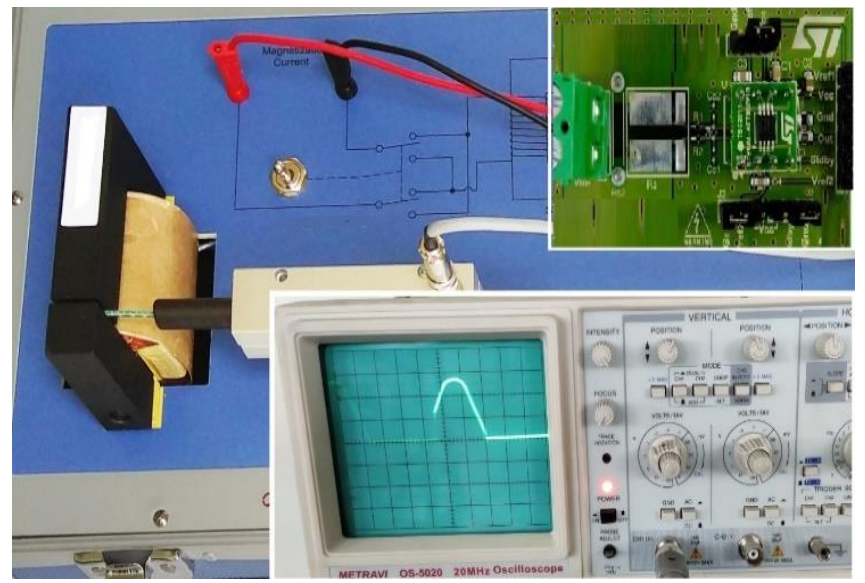

Fig. 5. Experimental setup with single-phase transformer showing the opamp integrator and the data acquisition system.

The technique used here for storage and curve fitting is piecewise mixed model [21]. In this technique, magnetization characteristics of the transformer is found experimentally in laboratory. The curve is then broken into piecewise mixed model for storage. The data for the curve is then compared with standard data and the inference about the operating condition is made for the difference data. For sinusoidal supply, the magnetization curve is shown in Fig.6. From the figure, it is seen that the magnetization curve is linear up to some extent and non-linear afterwards. It can be thus broken in to different small linear sections for storage. Afterwards, a mathematical analysis with the standard magnetization characteristics can be made. 

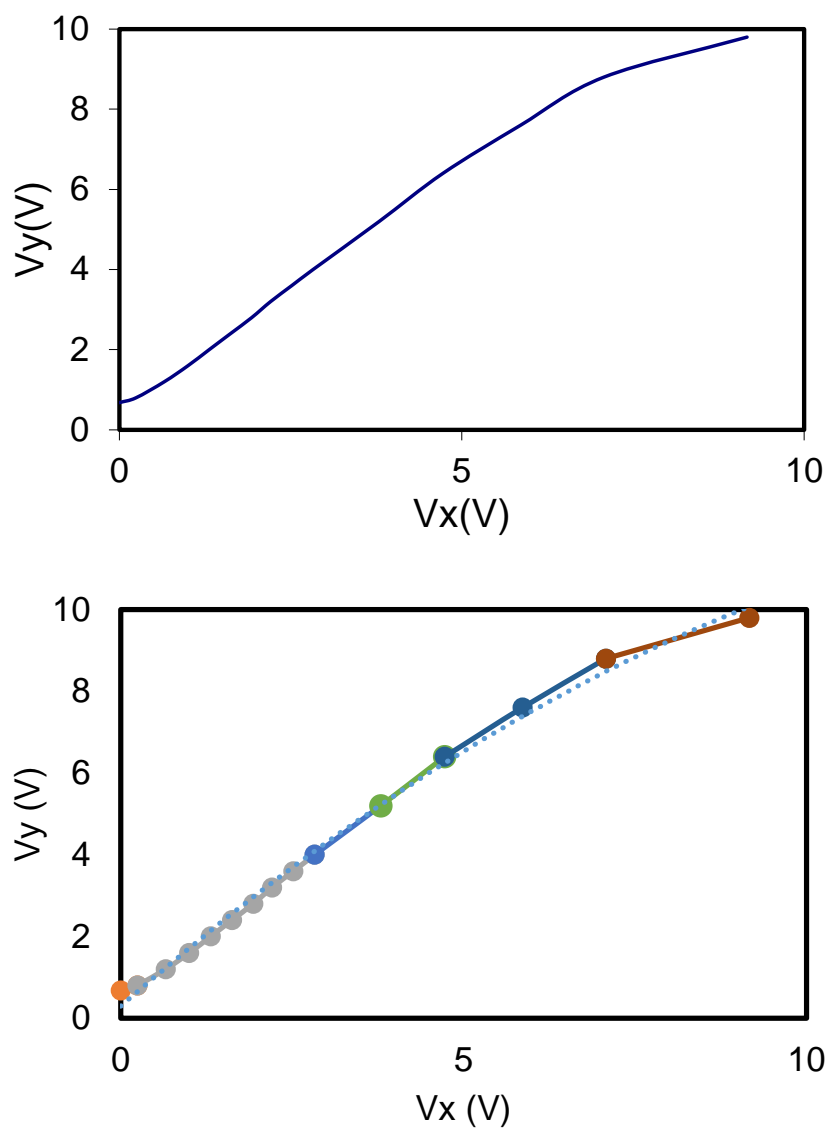

Fig. 6. Magnetization characteristics with the characteristics broken into piecewise mixed model with the trendline.

Fig.6 also indicates that the curve can be broken into small linear segments for storing purpose and later comparisons with a standard magnetization characteristic can be made later on. Similarly, the hysteresis plot can be stored as a whole.
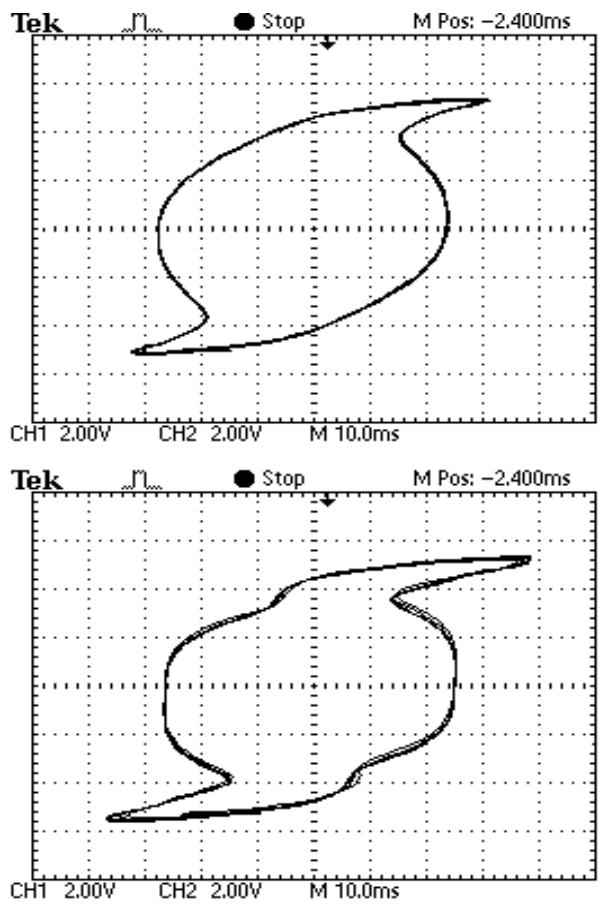

Fig.7. Experimental hysteresis curve for transformer core without source harmonics and with source harmonics of $3^{\text {rd }}$ and $5^{\text {th }}$ order respectively.
Fig.7 shows the Lissajous pattern of hysteresis curve obtained from experimental setup for source without any harmonics and for source with $3^{\text {rd }}$ and $5^{\text {th }}$ harmonics respectively. The magnetization curve is stored offline in Atmega microcontroller memory. This magnetization data attained is then compared with magnetization data without source harmonics. For comparison purpose, a simple subroutine is generated which tracks the changes in the attained data for a high deviation. If the deviated data is greater than a trivial tolerance value, then the transformer should be used in derated mode for reduced losses. The subroutine flowchart for comparison is shown in Fig.8.

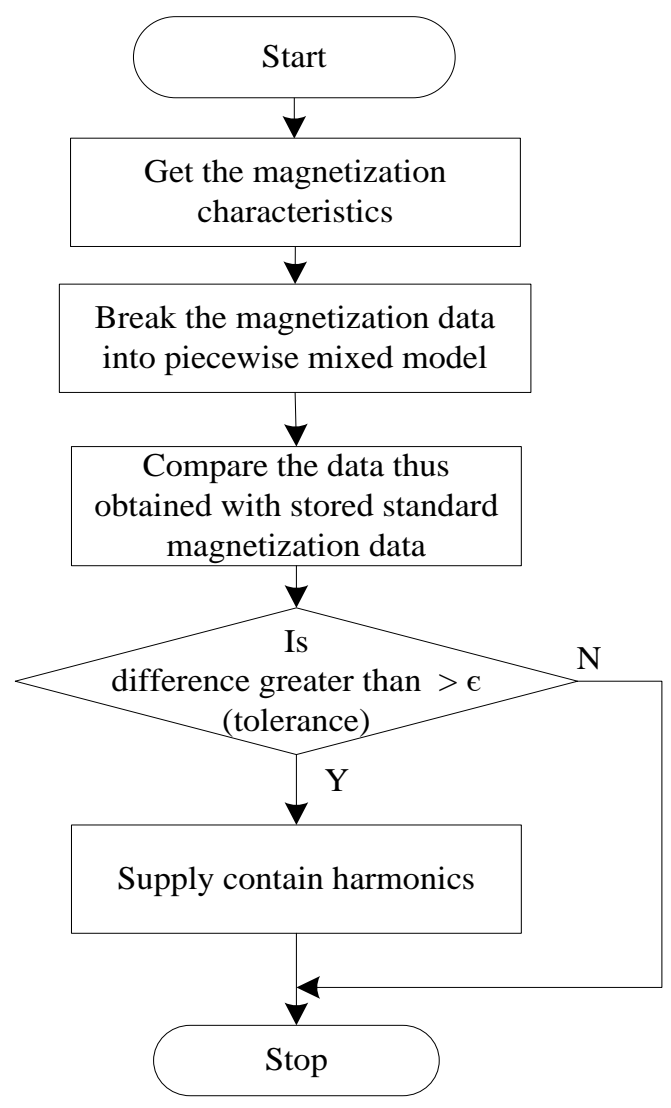

Fig.8. Flowchart for comparison and operation.

\section{CONCLUSION}

This paper presents an effective method to study and inspect the effect of source side harmonics on transformer operation. In this study, the effect of source side harmonics is observed on the hysteresis curve of the transformer core which can be used at the output of an inverter. The study also proposes a modified hysteresis-loss model for the transformer considering the effect of harmonics. The model can accurately predict the hysteresis loss as shown. Also, a new technique is presented for handy measurement of transformer internal parameters for plotting the hysteresis curve even when the transformer is under operation. The proposed study can be an effective tool for easy measurement and analysis of harmonic effects on the transformer. With increased source harmonics, the transformer can be operated in derated mode to reduce the winding currents and increase the operating life of the transformer reducing the losses. In future, a closed loop control can be added for increased accuracy of control. 
MATLAB/Simulink based simulations backed by suitable experiments validate the proposed study.

\section{REFERENCES}

[1] T.Dao, and B. T. Phung, "Effects of voltage harmonic on losses and temperature rise in distribution transformers," IET Generation Transmission and Distribution, vol. 12, no.2, pp. 347-354, Jan. 2018.

[2] V. Ioniţă, E. Cazacu and L. Petrescu, "Effect of voltage harmonics on iron losses in magnetic cores with hysteresis," 2018 18th International Conference on Harmonics and Quality of Power (ICHQP), Ljubljana, 2018, pp. 1-5.

[3] T. Dao, H. A. Halim, Z. Liu and B. T. Phung, "Voltage harmonic effect on losses in distribution transformers," 2016 International Conference on Smart Green Technology in Electrical and Information Systems (ICSGTEIS), Bali, 2016, pp. 27-32.

[4] E. So, R. Arseneau, and E. Hanique, "No-load loss measurements of power transformers under distorted supply voltage waveform conditions," IEEE Transactions on Instrumentation and Measurement, vol. 52, no. 2, pp. 429-432, Apr. 2003.

[5] J. Lavers, P. Biringer and H. Hollitscher, "Estimation of core losses when the flux waveform contains the fundamental plus a single odd harmonic component," IEEE Transactions on Magnetics, vol. 13, no. 5, pp. 1128-1130, September 1977.

[6] Y. Zhang, P. Pillay, M. Ibrahim and M. Cheng, "Magnetic characteristics and core losses in machine laminations: high-frequency loss prediction from low-frequency measurements," IEEE Transactions on Industry Applications, vol. 48, no. 2, pp. 623-629, March-April 2012,

[7] E. Arslan, S. Sakar and M. E. Balci, "On the no-load loss of power transformers under voltages with sub-harmonics," 2014 IEEE International Energy Conference (ENERGYCON), Cavtat, 2014, pp. 228-233.

[8] M. Amar and R. Kaczmarek, "A general formula for prediction of iron losses under nonsinusoidal voltage waveform," IEEE Transactions on Magnetics, vol. 31, no. 5, pp. 2504-2509, Sept. 1995.

[9] T. Nag, S. B. Santra, A. Chatterjee, D. Chatterjee and A. K. Ganguli, "Fuzzy logic-based loss minimisation scheme for brushless DC motor drive system," IET Power Electronics, vol. 9, no. 8, pp. 1581-1589, 2016.

[10] J. Faiz, M. Ghazizadeh and H. Oraee, "Derating of transformers under non-linear load current and non-sinusoidal voltage - an overview," IET Electric Power Applications, vol. 9, no. 7, pp. 486-495, 2015.

[11] D.G. Ece and H. Ackay, "An analysis of transformer excitation current under nonsinusoidal supply voltage," IEEE Transactions on Instrumentation and Measurement, vol. 51, no. 5, pp. 1085-1089, 2002.

[12] R. Arseneau, E. So and E. Hanique, "Measurements and correction of no-load losses of power transformers," IEEE Transactions on Instrumentation and Measurement, vol. 54, no. 2, pp. 503-506, 2005.

[13] T.D. Kefalas and A.G. Kladas, "Harmonic impact on distribution transformer no-load loss," IEEE Transactions on Industrial Electronics, vol. 57, no. 1, pp. 193-200, Jan. 2010.

[14] X. Liu, C. Yao, S. Liang, J. Wang and T. Liu, "Measurement of the noload characteristics of single-phase transformer using an improved low-frequency method," IEEE Transactions on Industrial Electronics, vol. 65, no. 5, pp. 4347-4356, May 2018.

[15] E. Purcell and D. Morin, "Electricity and Magnetism," New York: Cambridge University Press, 3rd ed., p. 278, 2013.

[16] W. Hayt, "Engineering Electromagnetics," McGraw-Hill, UK, $5^{\text {th }}$ ed., p. 312, 1989.

[17] S.D. Sudhoff, "Power Magnetic Devices: A Multi-Objective Design Approach,” John Wiley and Sons, USA, pp. 168-169, 2014.

[18] A. Chatterjee, K. Roy and D. Chatterjee, "A gravitational search algorithm (GSA) based photo-voltaic (PV) excitation control strategy for single phase operation of three phase wind-turbine coupled induction generator," Energy, vol.74, pp.707-718, 2014.

[19] M.R. Patel, "Wind and solar power systems: Design, analysis and operation," $2^{\text {nd }}$ ed., CRC Press, USA, 2006.

[20] A. Chatterjee and D. Chatterjee, "Analysis and control of photovoltaicassisted three-phase induction machine operating as single-phase micro-wind generator," IET Generation Transmission and Distribution vol. 10, no. 9, pp. 2165-2176, 2016.

[21] D. Chatterjee, "A novel magnetizing-curve identification and computer storage technique for induction machines suitable for online application," IEEE Transactions on Industrial Electronics, vol. 58, no. 12, pp. 5336-5343, Dec. 2011 